\title{
The germline variants in DNA repair genes in pediatric medulloblastoma: a challenge for current therapeutic strategies
}

Joanna Trubicka ${ }^{1,2^{*}}$, Tomasz Żemojtel ${ }^{3,4}$, Jochen Hecht ${ }^{5,6}$, Katarzyna Falana $^{1}$, Dorota Piekutowska- Abramczuk', Rafał Płoski , Marta Perek-Polnik ${ }^{8}$, Monika Drogosiewicz ${ }^{8}$, Wiesława Grajkowska ${ }^{2,9}$, Elżbieta Ciara ${ }^{1}$, Elżbieta Moszczyńska ${ }^{10}$, Bożenna Dembowska-Bagińska ${ }^{8}$, Danuta Perek ${ }^{8}$, Krystyna H. Chrzanowska ${ }^{1}$, Małgorzata Krajewska-Walasek ${ }^{1}$ and Maria Łastowska ${ }^{2,9}$

\begin{abstract}
Background: The defects in DNA repair genes are potentially linked to development and response to therapy in medulloblastoma. Therefore the purpose of this study was to establish the spectrum and frequency of germline variants in selected DNA repair genes and their impact on response to chemotherapy in medulloblastoma patients.

Methods: The following genes were investigated in 102 paediatric patients: MSH2 and RAD50 using targeted gene panel sequencing and NBN variants (p.I171V and p.K219fs*19) by Sanger sequencing. In three patients with presence of rare life-threatening adverse events (AE) and no detected variants in the analyzed genes, whole exome sequencing was performed. Based on combination of molecular and immunohistochemical evaluations tumors were divided into molecular subgroups. Presence of variants was tested for potential association with the occurrence of rare life-threatening AE and other clinical features.
\end{abstract}

Results: We have identified altogether six new potentially pathogenic variants in MSH2 (p.A733T and p.V606l), RAD50 (p.R1093*), FANCM (p.L694*), ERCC2 (p.R695C) and EXO1 (p.V738L), in addition to two known NBN variants. Five out of twelve patients with defects in either of MSH2, RAD50 and NBN genes suffered from rare life-threatening $\mathrm{AE}$, more frequently than in control group $(p=0.0005)$. When all detected variants were taken into account, the majority of patients (8 out of 15 ) suffered from life-threatening toxicity during chemotherapy.

Conclusion: Our results, based on the largest systematic study performed in a clinical setting, provide preliminary evidence for a link between defects in DNA repair genes and treatment related toxicity in children with medulloblastoma. The data suggest that patients with DNA repair gene variants could need special vigilance during and after courses of chemotherapy.

Keywords: Medulloblastoma, DNA repair genes, Toxicity

\footnotetext{
* Correspondence: j.trubicka@ipczd.pl

'Department of Medical Genetics, The Children's Memorial Health Institute,

Al. Dzieci Polskich 20, 04-730 Warsaw, Poland

${ }^{2}$ Department of Pathology, The Children's Memorial Health Institute, Al.

Dzieci Polskich 20, 04-730 Warsaw, Poland

Full list of author information is available at the end of the article
} 


\section{Background}

Brain tumors represent the leading cause of childhood cancer mortality. The most common malignant brain tumor among them is medulloblastoma [1]. Although multimodality treatment regimens have substantially improved survival in this disease, up to $30-40 \%$ of patients with medulloblastoma still die of the disease. Detrimental effect of current treatment on long-term survivors is also observed [2]. Our understanding of the molecular background of pediatric brain tumors has expanded significantly over the past few years. The vast amount of genomic and molecular data generated recently has proved that medulloblastoma is not a single entity but is composed of at least four subtypes: Wingless (WNT), Sonic Hedgehog (SHH), Group 3 and Group 4 (nonWNT/SHH types), with distinct genetic and biological profiles as well as different course of disease requiring adequate therapeutic approaches [2-6]. Despite of improved understanding of the molecular basis of medulloblastoma, many cases still lack an obvious genetic driver $[4,7,8]$. The further research focused on additional potential mechanisms responsible for the development of this tumor may lead to identification of new susceptibility factors as well as new markers that predict response to therapeutic agents and provide prognostic information. So far, majority of driver mutations detected in medulloblastoma are of somatic character. Impact of germline genetic variability that may affect clinicopathologic presentation of this tumor have not been in-depth investigated yet $[5,7,9-12]$.

In our study we focused on evaluation of germline defects in genes that play a role in DNA repair pathway because of the following reasons. Firstly, DNA-repair deficiency is associated with cancer development and the key role of germline alterations in promoting tumorigenesis is highlighted by several cancer predisposition syndromes e.g. Li-Fraumeni, Fanconi anemia or Turcot syndrome, where occurrence of medulloblastoma has been recorded. Secondly, it is well known that germline defects may modulate the response to treatment since DNA-repair mechanisms make cells prone to the effects of DNA-damaging chemotherapy [13-15]. It is important to notice that majority of evidence about the impact of DNA-repair genes defects on toxicity in medulloblastoma comes from either description of single cases $[16,17]$ or from mouse models and cell lines experiments $[13,18]$ but not from systemic clinical based investigation. Therefore, all these data indicate that DNA repair genes are a promising targets possibly linked both to development of tumor and response to therapy in medulloblastoma.

Within essential components of DNA repair signaling cascade the $N B N$ gene particularly draws attention as potentially susceptibility marker for medulloblastoma
$[19,20]$. Germline defects in medulloblastoma patients were observed also in other genes cooperated with $N B N$ in BRCA1-associated genome surveillance complex (BASC), including MSH6, PMS2 and MLH1 [21-24].

Biallelic defects in NBN gene result in Nijmegen Breakage Syndrome (NBS; OMIM:251,260), while homozygous defects in MSH6, PMS2 or MLH1 genes are molecular cause of Constitutional Mismatch Repair Deficiency Syndrome (CMRDS; OMIM:276,300), hereditary disorder associated with increased risk of cancers including medulloblastoma [25]. Among other genes responsible for CMRDS is also MSH2 (ID:4436, MIM:609,309), one of the key factor of DNA mismatch repair system which recognizes and repairs mispaired or unpaired nucleotides resulted from DNA replication errors [25]. There is an evidence that germline $\mathrm{MSH} 2$ defects may predispose to primary early-onset CNS tumors, especially glioblastoma [26]. In addition, De Rosa et al. suggest that in some families with Turcot syndrome the coexistence of colorectal and childhood brain tumors may result from a complete MMR deficiency [27]. However, association between $\mathrm{MSH} 2$ defects and medulloblastoma was not evaluated yet.

A very similar phenotype to NBS was seen in patients with Nijmegen Breakage Syndrome-like Disorder (NBSLD - OMIM:613,078) caused by defects in the RAD50 gene (ID:10,111, MIM:604,040). This gene encodes the protein involved in DNA double-strand break repair, cell cycle checkpoint activation, telomere maintenance and meiotic recombination suggesting that molecular variants disrupting its function may lead to genome instability and carcinogenesis [28]. Furthermore, inactivation of proteins like RAD50 required for the homologous recombination machinery leads to defects in the nervous system development indicating that components of this system can play crucial role in development and progression of various neuro-oncological diseases [29]. The frequency of the molecular variants in $R A D 50$ gene was, similarly to $\mathrm{MSH} 2$, not determined in medulloblastoma patients up to now.

Therefore the first purpose of this study was to establish the spectrum of germline defects in $M S H 2$ and $R A D 50$ genes, as well as frequency of two known $N B N$ variants in 102 patients with medulloblastoma. In the next step we have evaluated the hypothesis that DNA repair genes may affect a response to therapy in medulloblastoma patients. We have found that alterations in a range of DNA repair genes are associated with occurrence of rare severe adverse effects during chemotherapy in patients.

\section{Methods}

Patients and controls

A set of 102 medulloblastoma patients treated between 2004 and 2014 in the Neurosurgery and Oncology Departments of the Children's Memorial Health Institute (CMHI) 
in Warsaw, Poland were investigated in this study. Based on a combination of genomic and immunohistochemical (IHC) analyses, patients were divided into molecular subgroups (see methods). Presence of metastases at diagnosis was classified according to Chang et al. [30]. The clinical characteristics of the study cohort is outlined in Table 1.

To estimate the population frequency of detected MSH2 and RAD5O variants (independently of the data deposited in the public databases) the populationspecific control group was assembled. DNA samples from 300 healthy donors with negative cancer family history and sex matched to the patients' groups were collected. To exclude potential bias between adult and childhood population the control group consisted of donors age matched to the study group.

\section{Methods}

A total DNA was extracted from peripheral blood and tumors samples by use of the automatic magnetic bead-based (MagnaPure, Roche) and phenol/chloroform methods, respectively [31].

Table 1 The characteristics of 102 patients with medulloblastoma

\begin{tabular}{|c|c|c|}
\hline \multicolumn{2}{|l|}{ Medulloblastoma patients } & \multirow{2}{*}{$\begin{array}{l}\text { Number of patients } \\
14\end{array}$} \\
\hline Age at diagnosis (years) & $\leq 3$ & \\
\hline & $3-13$ & 88 \\
\hline \multirow[t]{2}{*}{ Gender } & Male & 66 \\
\hline & Female & 36 \\
\hline \multirow[t]{5}{*}{ Histologic type } & Classic & 69 \\
\hline & LCA & 16 \\
\hline & $\mathrm{D} / \mathrm{N}$ & 6 \\
\hline & MBEN & 6 \\
\hline & MBL & 5 \\
\hline \multirow[t]{3}{*}{ Metastasis } & NO & 70 \\
\hline & YES & 31 \\
\hline & NA & 1 \\
\hline \multirow[t]{6}{*}{ Molecular tumor subtype } & WNT & 11 \\
\hline & $\mathrm{SHH}$ & 9 \\
\hline & Non-WNT/SHH & 25 \\
\hline & Group 3 & 7 \\
\hline & Group 4 & 24 \\
\hline & NA & 26 \\
\hline \multirow[t]{4}{*}{ Treatment protocol } & $H R$ & 60 \\
\hline & $S R$ & 29 \\
\hline & $<3 \mathrm{yrs}$ & 13 \\
\hline & TOTAL & 102 \\
\hline
\end{tabular}

Abbreviations: $L C A$ large cell/anaplastic, $D / N$ desmoplastic/nodular, $M B E N$ with extensive nodularity, MBL medulloblastoma, subtype not known, NA not available, PPNG Polish Pediatric Neurooncology Group, HR -PPNG High Risk protocol, SR -PPNG Standard Risk protocol, <3 yrs. - PPNG protocol for children younger than 3 years old
To evaluate the sequence of $M S H 2$ and $R A D 50$ genes in 102 patients with medulloblastoma targeted gene panel sequencing was used. The $N B N$ c.511A $>\mathrm{G}$ and c.657_661del5 variant status was determined upon previously described conditions [19]. In three patients with severe adverse events after the chemotherapy but with no variants detected in $M S H 2, R A D 50$, and $N B N$ genes whole exome sequencing (WES) was carried out to explore possible defects in other DNA repair genes.

\section{Targeted gene panel sequencing}

For generation of the targeted amplicon libraries the Ion AmpliSeq ${ }^{\text {ma }}$ Custom 3G-Panelv2 (275 bp; Life Technologies Corporation; Carlsbad, CA, USA) consisting of 82 primer pairs to target all exons of the MSH2 and RAD50 genes (RefSeq:NM_000251.2 and RefSeq: NM_005732.3, respectively) was used. Polymerase chain reaction (PCR) was performed according to the manufacturer's recommendations with the Ion AmpliSeq ${ }^{\text {Tm }}$ Library Kit 2.0. Amplicon size distribution and library concentration was determined using Agilent DNA 1000 Kit (Agilent Technologies; Inc., Waldbronn, Germany). The final concentration of the sample pool was measured by Qubit dsDNA BR Assay Kit (Life Technologies Corporation; Carlsbad, CA, USA). Emulsion PCR and sequencing was performed on an Ion PGM Sequencer (Life Technologies Corporation; Carlsbad, CA, USA) using 318 Chips and the Ion PGM 200 Sequencing Kit according to the manufacturer's instructions. The sequence reads were mapped to the haploid human reference genome (hg19) with Novoalign (Novocraft Technologies). SNVs and short insertions and deletions (indels) were called using GATK version 2.8. [32] Variant annotation was performed with Jannovar [33].

\section{Whole exome sequencing}

WES Library preparation was performed using Nextera Rapid Capture Exome kit (Illumina). The samples were run on $1 / 4$ of lane each on HiSeq 1500 using $2 \times 75$ bp pairedend reads. After initial processing by the CASAVA, the generated reads were aligned to the hg19 reference genome with Burrows-Wheeler Alignment Tool and further processed by Genome Analysis Toolkit [32]. Base quality score recalibration, indel realignment, duplicate removal and the SNP/INDEL calling were done as described [34]. The detected variants were annotated using Annovar5 [35].

\section{Selection and validation of candidate variants}

A way of prioritizing variants was based on three main filtration steps, as follows:

1/.variants with the global minor allele frequency $(\mathrm{MAF}) \geq 0.01$ (either in the 1000 Genomes Project, ExAC Databases or in a matched control group) were filtered out; 
2/.nonsynonymous SNPs, coding sequence insertion/ deletions (indels), and canonical splice-site variants were selected;

3/.all variants occurring within DNA repair genes detected by WES were prioritized;

4/.variants annotated as deleterious by three and more prediction algorithms (PolyPhen-2, SIFT, Mutation Taster and FATHMM), nonsense mutations and deletions that introduce premature stop codons were classified as likely pathogenic and selected into further consideration. Functional consequences of splice variants were predicted by Human Splicing Finder, Splice Site Finder-like and MaxEntScan.

For all selected variants the amino acid position in functional domains and posttranslational modifications were verified using NCBI Protein [36] and Alamut-2.4-6 Software (Interactive Biosoftware; Rouen, France). Their contribution in carcinogenesis was verified in the Catalogue of Somatic Mutations (COSMIC database) [37], Human Gene Mutation Database (HGMD) [38], ClinVar database and Online Mendelian Inheritance in Man (OMIM). Correlation with the clinical features and the course of disease was assessed for each candidate variant.

\section{Variant validation}

Next generation sequencing results were validated using Sanger sequencing. Specific primers for PCR reactions are available upon request. PCR products were sequenced in 3130 Genetic Analyzer and evaluated with Sequencing Analysis Software v.5.4 (Applied Biosystems/ Life Technologies; Foster City, CA). The frequency of validated variants were evaluated in the matched control group in the same conditions. Additionally, prioritized variants in MSH2 and RAD50 were cross-checked with the parents' sequence data to identify inherited versus de novo changes. In four out of five carriers of MSH2, RAD50, FANCM or EXO1 gene variants tumour tissues were available and the presence of identified changes (p.V606I, p.R1093*, p.L694* and p.V738L) were verified.

\section{Determination of molecular subgroups in medulloblastoma patients}

Tumors included in the study were divided into the following molecular groups:

1/.WNT Group defined by the presence of at least two features as recommended by the International Medulloblastoma Working Group [2]: CTNNB1 pathogenic variant, immunohistochemical positive nuclear reaction against $\beta$-catenin (DB \#610154, $1: 800)$ and the presence of chromosome 6 monosomy. The screening test for CTNNB1 variant analysis and chromosome 6 monosomy were performed according to methods described previously [39].

2/.SHH Group defined by the presence of immunohistochemical positive reaction with anti-GAB1 (Abcam \#ab27439 and/or \#59362, 1:100) and anti-YAP1 (Santa Cruz \#sc-101,199, 1:50) antibodies [3].

3/.Non-WNT/SHH type (Group 3 or Group 4) included the remaining tumors, tested negative for the above features.

4/.For further discrimination of non-WNT/SHH tumours (in order to identify Group 3 or Group 4) in patients with detected DNA repair genes variants we applied NanoString's nCounter System analysis (NanoString Technologies, Seattle, USA). For identification of clusters a series of 48 medulloblastoma tumours were analized. Overall 30 non-WNT/SHH tumours from this study were evaluated. Total RNA was extracted from frozen or FFPE tumours using RNeasy kits (Qiagen). RNA integrity was assessed using an Agilent 2100 bioanalyzer. For four Groups assignment NanoString CodeSet of 22 genes has been applied as described by Northcott et al. [40] Hybridization to the probes was performed in NanoString Technologies, Seattle, USA. Data were normalized and samples were clustered using nSolver 2.5 software.

\section{Treatment complications assessment}

Treatment related complications data were retrospectively re-analyzed and assessed according to The Common Toxicity Criteria (CTC), version 4.0. [41] For comparative analyses only rare life-threatening adverse events of grade 4 (grade 5 was absent in our cohort) have been taken into account since they are the most challenging complications in clinical practice. The significance of assessed adverse events frequency and other clinical features in groups of patients with presence vs. absence of DNA repair genes candidate variants were calculated using the Fisher Exact test.

\section{Results}

\section{$M S H 2$ and $R A D 50$ analysis}

The analysis of $M S H 2$ and RAD50 coding sequences in 102 medulloblastoma patients revealed 53 germline variants in total. Most of them $(48 / 53 ; 90.6 \%)$ were single nucleotide variants (SNV), while remaining (5/53; 9.4\%) were small indels (Additional file 1: Table S1). The number of identified variants ranged from 2 to 16, an average of 7 variants per sample. After the filtration steps (three heterozygous candidate variants including c.1816G $>\mathrm{A}$ (p.V606I) and c.2197G >A (p.A733T) in MSH2, as well as c.3277C $>$ T (p.R1093*) in RAD50 were selected. All of them were uncommon $(1 / 102 ; 0.98 \%$ for each variant) in 
the patients group. None of them was observed in 1000 Genomes Project Database [42] as well as in the matched control group of 300 samples. Allele frequency of p.A733T MSH2 and p.R1093* RAD50 variants in ExAC Database were 0.000005 and 0.000001 , respectively. The MSH2 p.V606I variant in ExAC Database was not reported so far (Table 2).

Due to the lack or low frequency of candidate variants in control groups, the estimation of cancer risk associated with the presence of identified variants was not possible. DNA testing of probands' parents confirmed parental origin of all selected variants. The presence of identified changes in tumor tissue had been proven for MSH2 p.V606I and RAD50 p.R1093* variants. For MSH2 p.A733T variant this analysis was not possible because of the lack of tumor tissue.

Within the prioritized variants only p.R1093* in RAD50 gene (rs121912628) was reported as pathogenic in ClinVar database (OMIM: 604,040.0001) and in the COSMIC list of variants that have previously been associated with cancer predisposition (COSM1060699) [37].

\section{NBN c.511A>G and c.657_661del5 variants analysis}

In 102 patients with medulloblastoma six carriers of $N B N$ germline likely pathogenic variants $(\mathrm{c} .511 \mathrm{~A}>\mathrm{G}$ or c.657_661del5) were identified. Additional four NBN carriers reported in our previous study [19] were included in the analysis to increase a number of patients for assessment of clinical relevance of detected $N B N$ variants.

\section{Whole exome sequencing analysis}

In three patients with severe treatment complications but no presence of $M S H 2, R A D 50$ and NBN candidate variants the exome sequence was analyzed. In all cases rare candidate variants in genes essential for DNA repair pathway were detected, including p.L694* in FANCM (ID 57697, MIM:609,644), p.R695C in ERCC2 (ID 2068, MIM:126,340) and p.V738L in EXO1 (ID 9156, MIM:606,063). None of identified variants was reported in 1000 Genomes Database and in matched control group. Two variants p.R695C in ERCC2 and p.V738L in EXO1 were found in ExAC Database with frequency 0.000000012 and 0.00000086 , respectively. All detected variant were present in heterozygous state (Table 2).

\section{Characteristics of patients and tumors with molecular variants in DNA repair genes}

Distribution of clinical and biological features in patients with molecular variants in essential for DNA repair pathway genes is presented in Table 3.

There were no differences between groups of patients with presence vs. absence of either of candidate variants in MSH2, RAD50 and NBN genes in terms of age ( $<3$ years of age vs. $\geq 3$ years, not significant, n.s), gender (n.s), LCA pathology (n.s) and presence of metastases (M2 M3 vs. M0 M1, n.s). Tumours belonged to WNT Group (one tumour), Group 3 (one tumour) and Group 4 (8 tumours). Three tumours were not analyzed by NanoString method due to the lack of RNA but they belonged to non-WNT/SHH type (Group 3 or 4). In the remaining three cases molecular type was not determined due to the lack of tumor material. It has been noticed that none of the patients with $\mathrm{SHH}$ tumors had $M S H 2, R A D 50$ or NBN variants (9 patients analyzed) but these data did not reach statistical significance when compared to other groups (n.s).

Four out of 12 patients with MSH2, RAD50 and NBN molecular variants did not complete treatment protocol because of reduction of the dose of drugs or delays above 100 days due to presence of various degrees of adverse events. These included 4 out of 6 patients who died, therefore we refrained from examination of survival rates. Among them one NBN c.657_661del5 carrier died due to secondary leukemia 48 months after diagnosis.

To assess the potential impact of molecular defects on the course of treatment we recorded that three out of 9 patients with presence of $N B N$ variants and available clinical data, suffered from rare grade 4 adverse events during chemotherapy after the first course of treatment. These included central nervous system toxicity, pneumonia and colitis with gastrointestinal bleeding. For comparison, among 89 patients without molecular variants in none of NBN, MSH2 and RAD50 genes only three patients suffered from similar complications (enterocolitis grade 4, gastrointestinal bleeding grade 4 and central nervous system toxicity grade 4 ) despite an application of the same PPNG protocol (Additional file 2: Figure S1 A and B) and the difference was significant $(p=0.01)$. Moreover, two out of three patients with identified variants in $\mathrm{MSH} 2$ or RAD50 also displayed similar grade 4 complications, namely central nervous system toxicity and pneumonia. Therefore, 5 out of 12 patients with defects in either of $M S H 2, R A D 50$ and NBN genes suffered from rare grade 4 adverse events during chemotherapy and these combined results were even more significant when compared to the control group $(p=0.0005)$ than for $N B N$ gene alone. Among patients with detected defects in MSH2, RAD50 and NBN genes High Risk arm of the PPNG protocol (HR, Additional file 3: Table S2, Additional file 2: Figure S1 A and B) which included Cisplatin and Ifosfamide was not applied more frequently than in the series of patients from control group without detected defects in those genes $(p=0.13)$. When patients were subdivided according to treatment arm, the results pointing toward more frequent toxicity in patients with identified variants were also statistically significant $(p=0.01$ for HR arm only, and $p=0.01$ for protocol for children $<3$ years old only). 


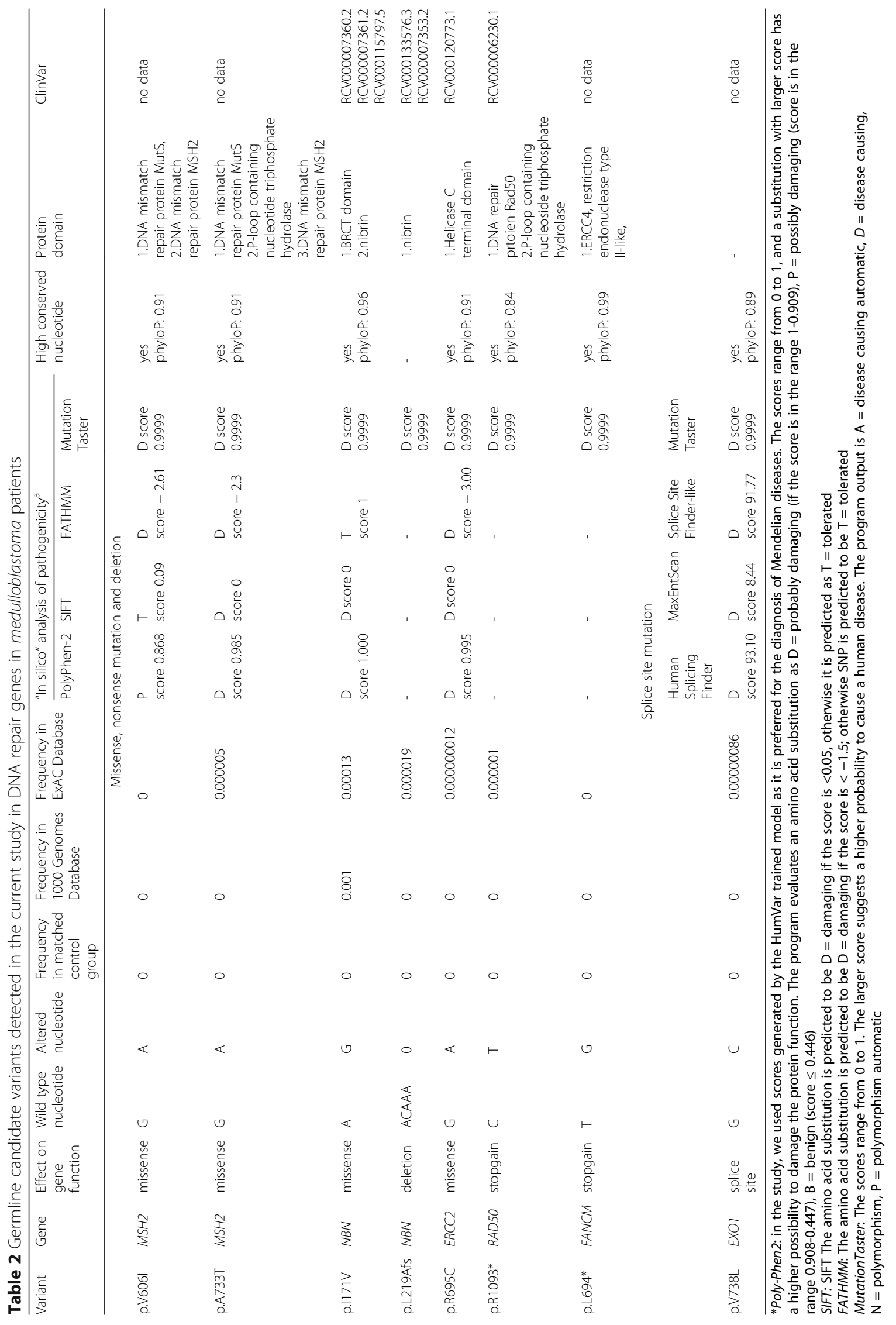




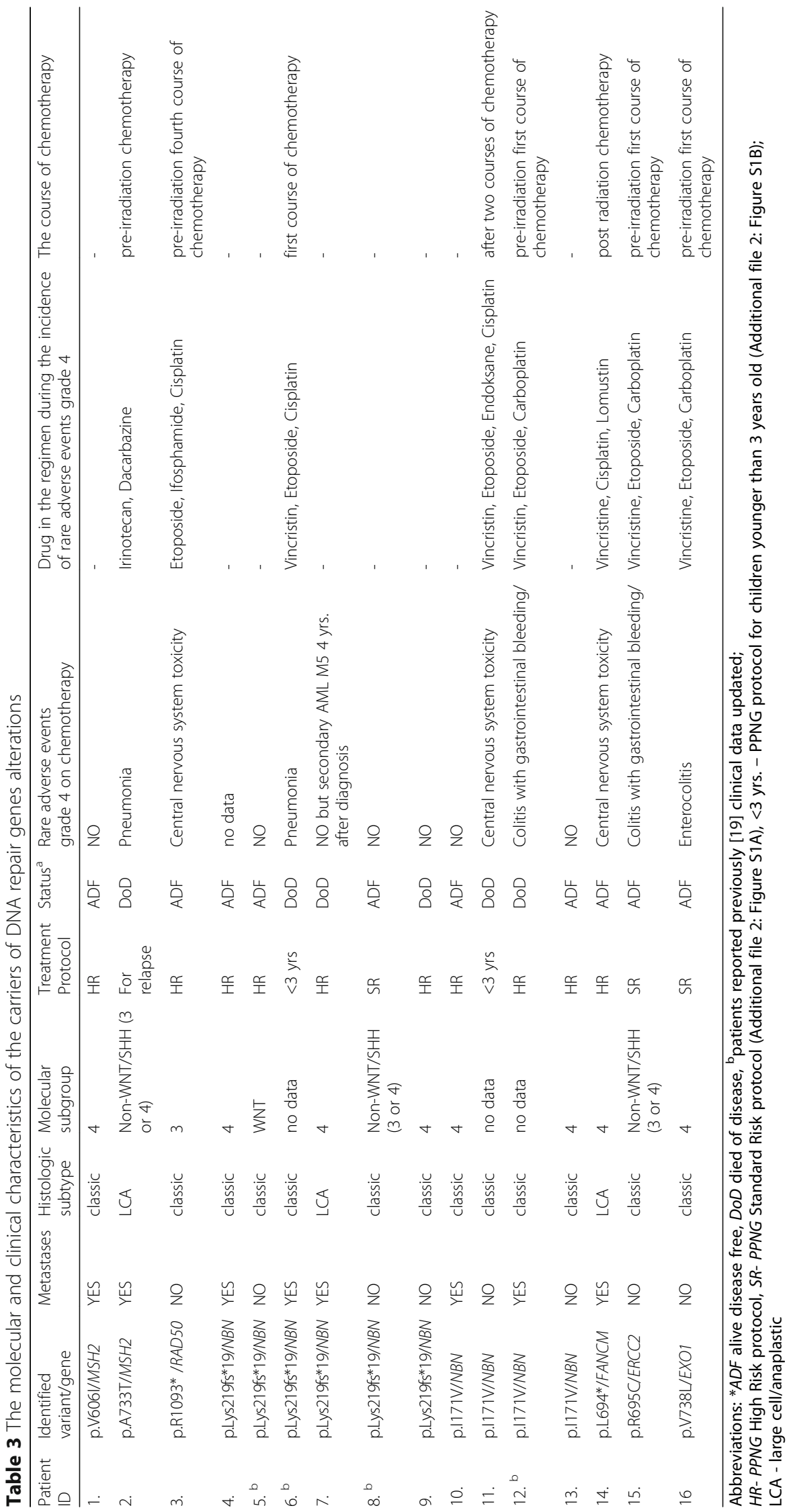


Because the above results indicate association between defects in analyzed DNA repair genes and presence of adverse effects during therapy we performed WES analyses in three patients who suffered from rare grade 4 adverse events but had no abnormalities in MSH2, RAD50 and $N B N$ genes. In all three patients candidate variants were detected in FANCM, ERCC2 and EXO1 genes which are presented in Tables 2 and 3. In summary, taking into account the WES results, among patients with detected variants in DNA repair genes majority of them (8 out of $15 ; 53 \%)$ suffered from rare life-threatening grade 4 toxicity during the course of treatment.

\section{Discussion}

Assuming that the fundamental feature of cancer is genomic instability, functional defects of proteins which are responsible for maintenance of genome integrity by correcting DNA replication errors, should be carcinogenic. It is therefore not surprising that a number of cancer susceptibility genes encode key factors of DNA repair pathways. Recent comprehensive analysis of germline mutations in pediatric cancers pointed to DNA repair genes as the most commonly mutated genes, including TP53 and $B R C A 2$ [43]. It is also increasingly clear that defects in DNA repair genes may determine patient's response to radio and chemotherapy $[13,16,17]$. In view of that we evaluated the potential association between DNA repair defects and treatment related toxicity as well as their potential role as a susceptibility factor for medulloblastoma.

The sequence analysis of two well-known repair genes MSH2 and RAD50 conducted in large cohort of 102 medulloblastoma patients revealed three new germline variants $M S H 2$ p.V606I and p.A733T as well as RAD50 p.R1093\%. Both the localization and the character of detected variants allow for prediction of their probably pathogenic impact on the encoded proteins what was supported by the results of the in silico analysis (Table 2). The p.V606I and p.A733T substitutions are localized in the crucial DNA mismatch repair protein V (MutSV - aa 619-854, pF00488) in highly (p.V606I- phyloP:4.40) and moderate (p.A733T-phyloP:2.55) conserved amino acid region. MutSV domain contains the dimerization interface and nucleotide-binding site with C-terminal helix-Uturn-helix motif that is critical for MutS function [44]. The RAD50 p.R1093* variant resulting in premature stop codon has severe consequences on the protein translation and predicts suppression of its protein. All identified variants were uncommon in our patients $(1 / 102 ; 0.98 \%)$. This is consistent with the published data indicating that molecular variants in $M S H 2$ and $R A D 50$ in CNS tumors occurred very rarely $(5 / 1637-0.31 \%$ and $4 / 1743-0.23 \%$, respectively [37]. In support of that, in published recently study of germline mutations in pediatric cancers, including medulloblastoma, MSH2 and RAD50 variants were not reported [43]. Due to the lack or low frequency of candidate variants in control groups the estimation of cancer risk associated with their presence was not possible (Table 2). However, deleterious character of detected germline variants, the role of the encoded proteins in DNA repair system and their annotation with genetic syndromes, including NBSLD and CMRDS associated with medulloblastoma, make them the potential susceptibility variants for this kind of tumor. The RAD50 p.R1093* variant was reported as one of two known molecular defects (HGMD CMO92910) responsible for NBSLD. In medulloblastoma patients pathogenic variants in MLH1, MSH6 and PMS2 genes were detected previously [21-24, 43]. Alterations in these genes together with $M S H 2$ defects lead to CMRDS. Both MSH2 and RAD50 encode the crucial components of the DNA repair system. $M S H 2$ belongs to mismatch repair genes (MMR) while RAD50 together with MRE11 and NBN constitute the MRN complex responsible for connecting DNA damage detection to DNA repair and cell cycle checkpoint function $[44,45]$. Biallelic deficiency in MMR genes had been referred as a molecular cause of increased predisposition to gastrointestinal and hematological malignances, as well as early-onset CNS tumors (especially glioblastoma; GBM) [22, 26]. Additionally, the germline heterozygous variants in MMR gene were reported in patients with Turcot syndrome associated with medulloblastoma incidence. The molecular variants affecting genes of the MRN complex might also play a role in pediatric tumor development. The evidence that NBN heterozygous variants predispose to childhood acute lymphoblastic leukemia and medulloblastoma was already published [19, 46-48]. All these facts reinforce potential role of DNA repair genes, including $M S H 2$ and $R A D 50$ in susceptibility to medulloblastoma but detailed mechanistic studies are required to confirm this preliminary hypothesis.

Notwithstanding the role of DNA repair genes in pathogenesis of medulloblastoma, it is profoundly important from the clinical perspective that the presence of molecular defects in these genes may have an impact on the course of treatment.

The MRN complex genes, including MRE11, NBN and $R A D 50$ are required for double-stand DNA break (DBS) repair via one of the DNA repair system, homologous recombination (HR). Defects in HR system lead to hypersensitivity to agents that produce DSB and topoisomerase inhibitors eg. etoposide $[14,15,49]$. MMR genes remove mispaired nucleotides by the cooperation in mismatch repair system whose defects are associated with hypersensitivity to DNA crosslinks and platinumbased chemotherapeutic agents (eg. mitomycin $\mathrm{C}$ and carboplatin) [14, 15, 49].

Medulloblastoma treatment protocol (Additional file 2: Figure S1 A and B) includes the drugs mentioned above, 
specifically platinum-based chemotherapeutic agents (carboplatin, cisplatin), topoisomerase inhibitor etoposide and, in addition, mitotic inhibitor vincristine. Therefore it is very likely that our patients with molecular variants in DNA repair genes may be more prone to complications in recovering from chemotherapy induced DNA damage. They include the patients with variants detected by WES analysis in ERCC2, FANCM or EXO1 genes, an essential components of DNA repair systems [14]. All three identified variants (p.R695C, p.L694* and p.V738L, respectively) were localized in highly conserved nucleotide position (phyloP: 0.89-0.99) in crucial for the encoded protein domains (Table 2) and the character of detected variants (nonsense and splice site) strengthens their pathogenic role. Previous functional studies had also linked these variants to increased sensitivity to therapeutic agents. Defects of ERCC2 protein were reported as a cause of faults in the nucleotide excision repair mechanism (NER) which is responsible for removal of variety of helix-distorting DNA lesions, as well as a hypersensitivity to platinum derivatives. The FANCM gene is one of the elements of the Fanconi Anemia (FANC) pathway responsible for DNA crosslinks repair, possibly through coordination of three main DNA repair systems: nonhomologus endjoing (NHEJ), homologus recombination (HR) and translesion DNA synthesis (TLS). Loss of function of this system results in sensitivity to DNA crosslinking agents and platinum derivatives [14, 15, 49]. Finally, EXO1 gene encode a nuclease which cooperates with MRN complex in DSB repair via $H R$ pathway as well as interacts with MMR genes in repair of DNA mismatches [50,51].

Although we acknowledge that functional studies are necessary to explore the mechanism through which DNA repair gene defects influence the treatment related toxicity we have already found significant association between defects in NBN, MSH2, RAD50, FANCM, ERCC2 and EXO1 genes and clinical data. Indeed, more than half of patients with variants in DNA repair genes suffered from rare adverse grade 4 events after administration of chemotherapy (Table 3). We acknowledge that validation cohorts would be necessary for confirmation of our results. Unfortunately, recently published NSG 'discovery sets' of medulloblastoma ranged only from 39 to 92 samples and molecular defect in MSH2, RAD50 and NBN gene were not identified [7, 9-12, 43]. Also, an information related to the therapy and accompanied side effects was not provided in these studies. However in two pediatric medulloblastoma patients with mutations in DNA repair genes (PALB2 and BRCA2) chemotherapy inducted grade 4 side effects were reported $[16,17]$. In addition, effect of other drugs being introduced to medulloblastoma treatment protocols e.g. temozolomide (TZM) may be dependent on the status of mismatch repair genes.
In melanoma one variant g.73170 $>\mathrm{C}$ in $\mathrm{MSH} 2$ gene (rs2303428) was associated with response and side effects and could be used as a molecular marker for TMZ treatment response [52].

On the other hand it is difficult to compare toxic effects caused by cancer treatment in adult patients harboring defects in DNA repair genes with toxicity observed in still developing and vulnerable tissues in children. Different spectrum of tumors in children and therefore different treatment protocols, including very high doses of drugs, may influence dissimilar reaction in the latter population.

\section{Conclusions}

Our study was conducted in a single institution on the largest series of uniformly treated patients which provided primary data indicating possible link between defects in DNA repair genes and treatment related toxicity in children. Given the complexity of the data in relation to the rarity of medulloblastoma, our results needs to be confirmed in independent cohorts. If proven, the special vigilance during and after treatment of patients with pathogenic variants in DNA repair genes should be required. Also additional screening for the presence of molecular variants in patients with manifestation of severe adverse events is necessary to acquire more information about their impact on the course of treatment. Finally, the potential revision of the mode of treatment should be considered for patients with germline defects in DNA repair genes in the future.

\section{Additional files}

Additional file 1: Table S1. The list of MSH2 and RAD50 gene germline variants detected in cohort of 102 MB patients. (DOC 92 kb)

Additional file 2: Figure S1A. Polish Pediatric Neurooncology Group (PPNG) treatment protocol for medulloblastoma patients (in children older than 3 years). Figure S1B. Polish Pediatric Neurooncology Group (PPNG) treatment protocol for medulloblastoma patients (in children younger than 3 years). (ZIP $575 \mathrm{~kb}$ )

Additional file 3: Table S2. The molecular and clinical characteristics of 102 patients with medulloblastoma. (XLSX $14 \mathrm{~kb}$ )

\footnotetext{
Abbreviations

$<3$ yrs.: Polish Pediatric Neurooncology Group protocol for children younger than 3 years old; ADF: Alive disease free; AE: Rare life-threatening adverse events; BASC: BRCA1-associated genome surveillance complex; CMHI: Childreñ's Memōorial Health Institutē in Warsaw, Poland; CMRDS: Constitutional Mismatch Repair Deficiency Syndrome; COSMIC database: Catalogue of Somatic Mutations; CTC: The Common Toxicity Criteria; D/N: Desmoplastic/nodular type of medulloblastoma; DBS: Doublestand DNA break repair; DoD: Died of disease; FANC: Fanconi Anemia; HGMD: Human Gene Mutation Database; HR: Homologous recombination; HR: Polish Pediatric Neurooncology Group High Risk protocol; IHC: Immunohistochemical analyses; Indels: Coding sequence insertion/ deletions; LCA: Large cell/anaplastic type of medulloblastoma; MAF: Minor allele frequency; MBEN: Medulloblastoma with extensive nodularity; MBL: Medulloblastoma, subtype not known; MMR: Mismatch repair genes; NBSLD: Nijmegen Breakage Syndrome-like Disorder; NER: Nucleotide excision repair mechanism; NHEJ: Nonhomologus endjoing; OMIM: Online Mendelian
} 
Inheritance in Man; PCR: Polymerase Chain Reaction; PPNG: Polish Pediatric Neurooncology Group; SHH: Sonic Hedgehog type of medulloblastoma; SR: Polish Pediatric Neurooncology Group Standard Risk protocol; TLS: Translesion DNA synthesis; WES: Whole Exome Sequencing; WNT: Wingless type of medulloblastoma

\section{Acknowledgments}

We thank the patients and their parents for the participation in this study. We would like to sincerely thank other colleagues from the Department of Oncology in the Children's Memorial Health Institute, especially: Iwona Filipek, Ewa Święszkowska, Maciej Balas, Magdalena Tarasińska, and Piotr Stawiński from Medical University of Warsaw for bioinformatics support. We are also grateful to Mrs. Ulrike Krüger from Institute for Medical Genetics and Human Genetics, Charité Universitätsmedizin Berlin as well as Mrs. Dorota Siestrzykowska and Mrs. Teresa Wojtasiak from the Department of Medical Genetics, CMHI for excellent technical assistance.

\section{Funding}

This work was supported by National Science Centre (6917/B/P01/2011/40; N N407 691,740 to JT, 2011/01/B/NZ4/01066: to MŁ), EU Structural Funds (Project POIG.02.01.00-14-059/09 to JT) and by Internal Funding from the Children's Memorial Health Institute, Warsaw, Poland (Grant no S124/2012 to $M\lfloor$ and $233 / 15$ to JT). None of these fundings participated in the design of the study and collection, analysis, and interpretation of data and in writing the manuscript.

\section{Availability of data and materials}

Complete results of WES including FASTQ files are be available in Sequence Read Archive (SRA) repository, (http://www.ncbi.nlm.nih.gov/bioproject/ 374506), BioProject ID:PRJNA374506, SubmissionID:SUB2397997. The full list of MSH2 and RAD50 gene germline variants detected in cohort of $102 \mathrm{MB}$ patients is presented in Additional file 1: Table S1.

\section{Authors' contributions}

JT and M $\mathrm{M}$ conceived and designed experiments. JT, KF, DPA, EC, TŻ, JH, RP performed experiments. TŻ, JH, JT, MŁ analyzed the data. MPP, MD, DP, BDB, recruited patients and provided clinical information. MKW, KCH provided clinical genetics evaluation of the patients. EM recruited persons to the control group. WG provided histopathological evaluation. JT, MŁ, DPA wrote the paper. KF, EM, MPP, MD, been involved in drafting the manuscript. BDB, MKW, KCH, RP, WG, EC critically reviewed the paper. All authors have read and approved the final version of the manuscript.

\section{Competing interests}

The authors declare that they have no competing interests.

\section{Consent for publication}

Not applicable.

\section{Ethics approval and consent to participate}

The study protocol was approved by the $\mathrm{CMHI}$ Bioethics Committee and written informed consent was obtained from the parents of the participants.

\section{Publisher's Note}

Springer Nature remains neutral with regard to jurisdictional claims in published maps and Institutional affiliations.

\section{Author details}

'Department of Medical Genetics, The Children's Memorial Health Institute, Al. Dzieci Polskich 20, 04-730 Warsaw, Poland. Department of Pathology, The Children's Memorial Health Institute, Al. Dzieci Polskich 20, 04-730 Warsaw, Poland. ${ }^{3}$ Institute for Medical Genetics and Human Genetics, Charité Universitätsmedizin Berlin, Augustenburger Platz 1, 13353 Berlin, Germany. ${ }^{4}$ Institute of Bioorganic Chemistry, Polish Academy of Sciences, 60-569 Poznan, Poland. ${ }^{5}$ Max Planck Institute for Molecular Genetics, Ihnestr. 63-73, 14195 Berlin, Germany. ${ }^{6}$ Berlin-Brandenburg Center for Regenerative Therapies, Charité Universitätsmedizin Berlin, 13353 Berlin, Germany. ${ }^{7}$ Department of Medical Genetics, Warsaw Medical University, Warsaw, Poland. ${ }^{8}$ Department of Oncology, The Children's Memorial Health Institute, Al. Dzieci Polskich 20, 04-730 Warsaw, Poland. ${ }^{9}$ Department of Experimental and Clinical Pathology, Mossakowski Medical Research Centre Polish
Academy of Sciences, A. Pawińskiego 5, 02-106 Warsaw, Poland.

${ }^{10}$ Department of Endocrinology and Diabetology, The Children's Memorial Health Institute, Al. Dzieci Polskich 20, 04-730 Warsaw, Poland.

Received: 10 February 2016 Accepted: 22 March 2017

Published online: 04 April 2017

\section{References}

1. Northcott PA, Rutka JT, Taylor MD. Genomics of medulloblastoma: from Giemsa-banding to next-generation sequencing in 20 years. Neurosurg Focus. 2010;28(1):E6.

2. Gottardo NG, Hansford JR, McGlade JP, Alvaro F, Ashley DM, Bailey S, Baker DL, Bourdeaut F, Cho YJ, Clay M, et al. Medulloblastoma down under 2013: a report from the third annual meeting of the international Medulloblastoma working group. Acta Neuropathol. 2014;127(2):189-201.

3. Ellison DW, Dalton J, Kocak M, Nicholson SL, Fraga C, Neale G, Kenney AM, Brat DJ, Perry A, Yong WH, et al. Medulloblastoma: clinicopathological correlates of SHH, WNT, and non-SHH/WNT molecular subgroups. Acta Neuropathol. 2011;121(3):381-96.

4. Kool M, Korshunov A, Remke M, Jones DT, Schlanstein M, Northcott PA Cho YJ, Koster J, Schouten-van Meeteren A, van Vuurden D, et al. Molecular subgroups of medulloblastoma: an international meta-analysis of transcriptome, genetic aberrations, and clinical data of WNT, SHH, group 3, and group 4 medulloblastomas. Acta Neuropathol. 2012;123(4):473-84.

5. Northcott PA, Shih DJ, Peacock J, Garzia L, Morrissy AS, Zichner T, Stutz AM, Korshunov A, Reimand J, Schumacher SE, et al. Subgroup-specific structural variation across 1,000 medulloblastoma genomes. Nature. 2012;488(7409):49-56.

6. Taylor MD, Northcott PA, Korshunov A, Remke M, Cho YJ, Clifford SC, Eberhart CG, Parsons DW, Rutkowski S, Gajjar A, et al. Molecular subgroups of medulloblastoma: the current consensus. Acta Neuropathol. 2012;123(4):465-72.

7. Jones DT, Jager N, Kool M, Zichner T, Hutter B, Sultan M, Cho YJ, Pugh TJ, Hovestadt V, Stutz AM, et al. Dissecting the genomic complexity underlying medulloblastoma. Nature. 2012;488(7409):100-5.

8. Northcott PA, Jones DT, Kool M, Robinson GW, Gilbertson RJ, Cho YJ, Pomeroy SL, Korshunov A, Lichter P, Taylor MD, et al. Medulloblastomics: the end of the beginning. Nat Rev Cancer. 2012;12(12):818-34.

9. Parsons DW, Li M, Zhang X, Jones S, Leary RJ, Lin JC, Boca SM, Carter H, Samayoa J, Bettegowda C, et al. The genetic landscape of the childhood cancer medulloblastoma. Science. 2011;331(6016):435-9.

10. Pugh TJ, Weeraratne SD, Archer TC, Pomeranz Krummel DA, Auclair D, Bochicchio J, Carneiro MO, Carter SL, Cibulskis K, Erlich RL, et al. Medulloblastoma exome sequencing uncovers subtype-specific somatic mutations. Nature. 2012:488(7409):106-10.

11. Rausch T, Jones DT, Zapatka M, Stutz AM, Zichner T, Weischenfeldt J, Jager N, Remke M, Shih D, Northcott PA, et al. Genome sequencing of pediatric medulloblastoma links catastrophic DNA rearrangements with TP53 mutations. Cell. 2012;148(1-2):59-71.

12. Robinson G, Parker M, Kranenburg TA, Lu C, Chen X, Ding L, Phoenix TN, Hedlund E, Wei L, Zhu X, et al. Novel mutations target distinct subgroups of medulloblastoma. Nature. 2012;488(7409):43-8.

13. Daniel RA, Rozanska AL, Mulligan EA, Drew Y, Thomas HD, Castelbuono DJ, Hostomsky Z, Plummer ER, Tweddle DA, Boddy AV, et al. Central nervous system penetration and enhancement of temozolomide activity in childhood medulloblastoma models by poly (ADP-ribose) polymerase inhibitor AG-014699. Br J Cancer. 2010;103(10):1588-96.

14. Kennedy RD, D'Andrea AD. DNA repair pathways in clinical practice: lessons from pediatric cancer susceptibility syndromes. J Clin Oncol. 2006:24(23):3799-808

15. Lambrechts $S$, Lambrechts $D$, Despierre E, Van Nieuwenhuysen E, Smeets D, Debruyne PR, Renard V, Vroman P, Luyten D, Neven P, et al. Genetic variability in drug transport, metabolism or DNA repair affecting toxicity of chemotherapy in ovarian cancer. BMC Pharmacol Toxicol. 2015;16:2.

16. Rube CE, Fricke A, Schneider R, Simon K, Kuhne M, Fleckenstein J, Graber S, Graf N, Rube C. DNA repair alterations in children with pediatric malignancies: novel opportunities to identify patients at risk for high-grade toxicities. Int J Radiat Oncol Biol Phys. 2010;78(2):359-69.

17. Miele E, Mastronuzzi A, Po A, Carai A, Alfano V, Serra A, Colafati GS, Strocchio L, Antonelli M, Buttarelli FR, et al. Characterization of 
medulloblastoma in Fanconi anemia: a novel mutation in the BRCA2 gene and SHH molecular subgroup. Biomark Res. 2015;3:13.

18. von Bueren AO, Bacolod MD, Hagel C, Heinimann K, Fedier A, Kordes U, Pietsch T, Koster J, Grotzer MA, Friedman HS, et al. Mismatch repair deficiency: a temozolomide resistance factor in medulloblastoma cell lines that is uncommon in primary medulloblastoma tumours. Br J Cancer. 2012;107(8):1399-408.

19. Ciara E, Piekutowska-Abramczuk D, Popowska E, Grajkowska W, Barszcz S, Perek D, Dembowska-Baginska B, Perek-Polnik M, Kowalewska E, Czajnska A, et al. Heterozygous germ-line mutations in the NBN gene predispose to medulloblastoma in pediatric patients. Acta Neuropathol. 2010;119(3):325-34.

20. Huang J, Grotzer MA, Watanabe T, Hewer E, Pietsch T, Rutkowski S, Ohgaki H. Mutations in the Nijmegen breakage syndrome gene in medulloblastomas. Clin Cancer Res. 2008;14(13):4053-8.

21. Scott RH, Mansour S, Pritchard-Jones K, Kumar D, MacSweeney F, Rahman N. Medulloblastoma, acute myelocytic leukemia and colonic carcinomas in a child with biallelic MSH6 mutations. Nat Clin Pract Oncol. 2007;4(2):130-4.

22. Roy S, Raskin L, Raymond VM, Thibodeau SN, Mody RJ, Gruber SB. Pediatric duodenal cancer and biallelic mismatch repair gene mutations. Pediatr Blood Cancer. 2009;53(1):116-20.

23. Lindsay H, Jubran RF, Wang L, Kipp BR, May WA. Simultaneous colonic adenocarcinoma and medulloblastoma in a 12-year-old with biallelic deletions in PMS2. J Pediatr. 2013;163(2):601-3.

24. Wang Q, Lasset C, Desseigne F, Frappaz D, Bergeron C, Navarro C, Ruano E, Puisieux A. Neurofibromatosis and early onset of cancers in hMLH1-deficient children. Cancer Res. 1999;59(2):294-7.

25. Familial Cancer Database. Available at www.familialcancerdatabase.nl/. Accessed 20 Dec 2014

26. Bougeard G, Charbonnier F, Moerman A, Martin C, Ruchoux MM, Drouot N, Frebourg T. Early onset brain tumor and lymphoma in MSH2-deficient children. Am J Hum Genet. 2003;72(1):213-6.

27. De Rosa M, Fasano C, Panariello L, Scarano MI, Belli G, lannelli A, Ciciliano F, Izzo P. Evidence for a recessive inheritance of Turcot's syndrome caused by compound heterozygous mutations within the PMS2 gene. Oncogene. 2000;19(13):1719-23.

28. Berardinelli F, di Masi A, Antoccia A. NBN Gene polymorphisms and cancer susceptibility: a systemic review. Current genomics. 2013;14(7):425-40.

29. Chrzanowska K, Trubicka J, Ciara E. Pediatric Medulloblastoma: the role of heterozygous germ-line mutations in the NBN Gene. In: Hayat MA, editor. Pediatric Cancer, Volume 3, vol. Volume 3. Netherlands: Springer; 2012. p. 117-27.

30. Chang $\mathrm{CH}$, Housepian EM, Herbert Jr C. An operative staging system and a megavoltage radiotherapeutic technic for cerebellar medulloblastomas. Radiology. 1969;93(6):1351-9.

31. Sambrook J, Maniatis T, Fritsch EF. Cold Spring Harbor laboratory:: molecular cloning : a laboratory manual, 2nd edn. Cold Spring Harbor, N.Y: Cold Spring Harbor Laboratory Press; 1989.

32. McKenna A, Hanna M, Banks E, Sivachenko A, Cibulskis K, Kernytsky A, Garimella K, Altshuler D, Gabriel S, Daly M, et al. The genome analysis toolkit: a MapReduce framework for analyzing next-generation DNA sequencing data. Genome Res. 2010;20(9):1297-303.

33. Jäger M, Wang K, Bauer S, Smedley D, Krawitz P, Robinson PN. Jannovar: a java library for Exome annotation. Hum Mutat. 2014;35(5):548-55.

34. DePristo MA, Banks E, Poplin R, Garimella KV, Maguire JR, Hart C, Philippakis AA, del Angel G, Rivas MA, Hanna M, et al. A framework for variation discovery and genotyping using next-generation DNA sequencing data. Nat Genet. 2011:43(5):491-8.

35. Wang K, Li M, Hakonarson H. ANNOVAR: functional annotation of genetic variants from high-throughput sequencing data. Nucleic Acids Res. 2010;38(16):e164.

36. NCBI Protein. Available at http://www.ncbi.nlm.nih.gov/protein. Accessed 20 Dec 2014.

37. COSMIC database. Available at http://cancer.sanger.ac.uk/cancergenome/ projects/cosmic/. Accessed 1 Dec 2014.

38. Human Gene Mutation Database. Available at http://www.hgmd.cf.ac.uk/ac/ index.php. Accessed 24 Nov 2014.

39. Łastowska M, Jurkiewicz E, Trubicka J, Daszkiewicz P, Drogosiewicz M, Malczyk K, Grajkowska W, Matyja E, Cukrowska B, Pronicki M, et al. Contrast enhancement pattern predicts poor survival for patients with non-WNT/SHH medulloblastoma tumours. J Neuro-Oncol. 2015;123(1):65-73.

40. Northcott PA, Shih DJ, Remke M, Cho YJ, Kool M, Hawkins C, Eberhart CG, Dubuc A, Guettouche T, Cardentey Y, et al. Rapid, reliable, and reproducible molecular sub-grouping of clinical medulloblastoma samples. Acta Neuropathol. 2012:123(4):615-26.
41. The Common Toxicity Criteria. Available at https://www.eortc.be/services/ doc/ctc/. In; Accessed 13 Nov 2015.

42. Genomes. A Deep Catalog of Human Genetic Variations. Available at http:// www.1000genomes.org. Accessed 15 Sept 2014.

43. Zhang J, Walsh MF, Wu G, Edmonson MN, Gruber TA, Easton J, Hedges D, Ma X, Zhou X, Yergeau DA, et al. Germline mutations in predisposition genes in pediatric cancer. N Engl J Med. 2015;373(24):2336-46.

44. Kunkel TA, Erie DA. DNA MISMATCH REPAIR*. Annu Rev Biochem. 2005; 74(1):681-710

45. Wang Y, Cortez D, Yazdi P, Neff N, Elledge SJ, Qin J. BASC, a super complex of BRCA1-associated proteins involved in the recognition and repair of aberrant DNA structures. Genes Dev. 2000;14(8):927-39.

46. Mosor M, Ziolkowska I, Pernak-Schwarz M, Januszkiewicz-Lewandowska D, Nowak J. Association of the heterozygous germline I171V mutation of the NBS1 gene with childhood acute lymphoblastic leukemia. Leukemia. 2006;20(8):1454-6.

47. Piekutowska-Abramczuk D, Ciara E, Popowska E, Grajkowska W, Dembowska-Baginska B, Kowalewska E, Czajnska A, Perek-Polnik M, Roszkowski M, Syczewska M, et al. The frequency of NBN molecular variants in pediatric astrocytic tumors. J Neuro-Oncol. 2010;96(2):161-8.

48. Chrzanowska KH, Piekutowska-Abramczuk D, Popowska E, Gladkowska-Dura M, Maldyk J, Syczewska M, Krajewska-Walasek M, Goryluk-Kozakiewicz B, Bubala H, Gadomski A, et al. Carrier frequency of mutation 657del5 in the NBS1 gene in a population of polish pediatric patients with sporadic lymphoid malignancies. Int J Cancer. 2006;1 18(5):1269-74.

49. Jackson SP, Bartek J. The DNA-damage response in human biology and disease. Nature. 2009;461(7267):1071-8.

50. Bolderson E, Tomimatsu N, Richard DJ, Boucher D, Kumar R, Pandita TK, Burma S, Khanna KK. Phosphorylation of Exo1 modulates homologous recombination repair of DNA double-strand breaks. Nucleic Acids Res. 2010;38(6):1821-31.

51. Rein K, Yanez DA, Terre B, Palenzuela L, Aivio S, Wei K, Edelmann W, Stark $J M$, Stracker TH. EXO1 is critical for embryogenesis and the DNA damage response in mice with a hypomorphic Nbs1 allele. Nucleic Acids Res. 2015:43(15):7371-87.

52. Boeckmann L, Schirmer M, Rosenberger A, Struever D, Thoms KM, Gutzmer R, Has C, Kunz M, Kuschal C, Laspe P, Schoen MP, Brockmoeller J, Emmert S. Effect of DNA repair host factors on temozolomide or dacarbazine melanoma treatment in Caucasians. Pharmacogenet Genomics. 2009 Oct;19(10):760-9.

\section{Submit your next manuscript to BioMed Central and we will help you at every step:}

- We accept pre-submission inquiries

- Our selector tool helps you to find the most relevant journal

- We provide round the clock customer support

- Convenient online submission

- Thorough peer review

- Inclusion in PubMed and all major indexing services

- Maximum visibility for your research

Submit your manuscript at www.biomedcentral.com/submit
Biomed Central 